\title{
New approximate analytical technique for the solution of time fractional fluid flow models
}

\author{
Umar Farooq ${ }^{1}$, Hassan Khan $^{1,2^{*}}$ (D, Fairouz Tchier ${ }^{3}$, Evren Hincal ${ }^{2}$, Dumitru Baleanu ${ }^{4,5}$ and \\ Haifa Bin Jebreen ${ }^{3}$
}

\author{
"Correspondence: \\ hassanmath@awkum.edu.pk \\ 1 Department of Mathematics, \\ Abdul Wali khan University Mardan \\ Mardan, Pakistan \\ ${ }^{2}$ Department of Mathematics, Near \\ East University TRNC, Mersin 10, \\ Turkey \\ Full list of author information is \\ available at the end of the article
}

\begin{abstract}
In this note, we broaden the utilization of an efficient computational scheme called the approximate analytical method to obtain the solutions of fractional-order Navier-Stokes model. The approximate analytical solution is obtained within Liouville-Caputo operator. The analytical strategy generates the series form solution, with less computational work and fast convergence rate to the exact solutions. The obtained results have shown a simple and useful procedure to analyze complex problems in related areas of science and technology.

Keywords: Approximate analytical method; Analytical solution; Caputo operator; Riemann-Liouville; Navier-Stokes model
\end{abstract}

\section{Introduction}

Recently, the old idea of fractional-order derivatives became the focal point for many researchers. The mathematicians investigated the recent applications of fractional derivatives in advanced fields of applied science and engineering. Since the fractional-order differential operator is nonlocal, the next state of a system depends on its current and previous states. The noninteger order derivatives have the major merit of describing the memory and heredity properties of various phenomena. Therefore the fractional-order derivatives and integrals have numerous applications in science and technology; for example, modeling nonlinear oscillations of earthquakes [1], the fractional-order fluid dynamic traffic model [2], chaos theory [3], signal processing phenomena [4], electrodynamics [5], fractional model of cancer chemotherapy [6], optics [7], fractional diabetes model [8], and other areas [9-14].

A famous viscous fluid flow equation of motion called the Navier-Stokes equation has been developed in 1822 [15]. This Navier-Stokes equation can be regarded as Newton's second law of motion for fluid substances, and it is a combination of the continuity, momentum, and energy equations. It describes many physical phenomena such as ocean currents, liquid flow in a pipe, blood flows in arteries, and airflow around aircraft wings.

(c) The Author(s) 2021. This article is licensed under a Creative Commons Attribution 4.0 International License, which permits use, sharing, adaptation, distribution and reproduction in any medium or format, as long as you give appropriate credit to the original author(s) and the source, provide a link to the Creative Commons licence, and indicate if changes were made. The images or other third party material in this article are included in the article's Creative Commons licence, unless indicated otherwise in a credit line to the material. If material is not included in the article's Creative Commons licence and your intended use is not permitted by statutory regulation or exceeds the permitted use, you will need to obtain permission directly from the copyright holder. To view a copy of this licence, visit http://creativecommons.org/licenses/by/4.0/. 
The Navier-Stokes model was first modeled as a fractional-order model by El-Shahed and Salem [16] in 2005 from the generalized classical NS equations.

Numerical and approximate analytical solutions of classical NSE and fractional NSE are the primary consideration of researchers. Different techniques have were used by researchers such as Kumar et al. [17], who solved a nonlinear fractional model of NS analytically by coupling LTA and HPM, Kumar et al. [18] also obtained an approximate solution of NSE by coupling ADM and LTA, Ganji et al. [19] and Ragab et al. [20] used HAM for solving fractional-order NS model. Rasool Shah et al. [21] applied a new hybrid method known as the Laplace-Adomian decomposition method (LADM) for analytical approximation of fractional-order system of NS equation. Momani and Odibat [22] used ADM for analytical solution of fractional-order NSE, and Birajdar [23] also applied ADM for approximate solution of fractional-order NSE. Chaurasia and Kumar [24] used the Laplace and Hankel transforms for analytical solution of fractional-order Navier-Stokes model, Parkash et al. [25] used q-HATM for analytical solution of the coupled fractional-order NS model, Barjes et al. [26] used FRDTM for numerical simulation of NSE. Some other recent numerical and analytical methods used for fractional-order models are [27-35].

In this paper, we develop the approximate analytical method (AAM) based on an analytical strategy using the Riemann-Liouville integral operator and Caputo operator (4). The analytical approximation is obtained by testing some linear and nonlinear NavierStokes models of fractional order. The AAM provided tentative results as compared to other numerical and approximate methods.

The rest of this paper is organized as follows. In Sect. 2, we present preliminary concepts. In Sect. 3, we present the procedure of AAM. In Sect. 4, we implement AAM on some fluid flow model. In Sect. 5, we conclude the results.

\section{Preliminaries and basic concepts}

In this section, we present the related definitions and preliminary concepts of fractional calculus and the procedure of the new AAM.

\subsection{Riemann-Liouville integral operator [36]}

The Riemann-Liouville fractional partial integral, denoted by $I_{t}^{\vartheta}$, where $\vartheta \in N, \vartheta \geq 0$, is defined as

$$
I_{t}^{\vartheta} v(\Im, t)= \begin{cases}\frac{1}{\Gamma(\vartheta)} \int_{0}^{t} v(\Im, t) d t, & \vartheta, t>0, \\ v(\Im, t), & \vartheta=0, t>0,\end{cases}
$$

where, $\Gamma$ is the gamma function.

\subsection{Some properties of Riemann- fractional partial integral}

Let $\vartheta, \beta \in R \backslash N, \vartheta, \beta>0, \rho>-1$. Then the operator $I_{t}^{\vartheta}$ has the following properties for functions $v(\Im, t)$ :

$$
\left\{\begin{array}{l}
I_{t}^{\vartheta} v(\Im, t) I_{t}^{\beta} v(\Im, t)=I_{t}^{\vartheta+\beta} v(\Im, t), \\
I_{t}^{\vartheta} v(\Im, t) I_{t}^{\beta} v(\Im, t)=I_{t}^{\beta} v(\Im, t) I_{t}^{\vartheta} v(\Im, t), \\
I_{t}^{\vartheta} t^{\rho}=\frac{\Gamma(\rho+1)}{\Gamma(\vartheta+\rho+1)} t^{\vartheta+\rho} .
\end{array}\right.
$$




\subsection{Caputo operator of fractional partial derivative [37]}

$$
D_{t}^{\vartheta} v(\Im, t)=\frac{\partial^{\vartheta} v(\Im, t)}{\partial t^{\vartheta}}= \begin{cases}I^{n-\vartheta}\left[\frac{\partial^{\vartheta} v(\Im, t)}{\partial t^{\vartheta}}\right], & n-1<\vartheta<n, n \in N, \\ \frac{\partial^{\vartheta} v(\Im, t)}{\partial t^{\vartheta}}, & n=\vartheta .\end{cases}
$$

Theorem 2.1 ([36]) Let $\vartheta, t \in R, t>0$, and $m-1<\rho<m \in N$. Then

$$
\begin{aligned}
& I_{t}^{\vartheta} D_{t}^{\vartheta} v(\Im, t)=v(\Im, t)-\sum_{k=0}^{m-1} \frac{t^{k}}{k !} \frac{\partial^{k} v\left(\Im, 0^{+}\right)}{\partial t^{k}}, \\
& D_{t}^{\vartheta} I_{t}^{\vartheta} v(\Im, t)=v(\Im, t) .
\end{aligned}
$$

\section{NAAM procedure for the solution of problems [36]}

In this section, we discuss a general procedure of NAAM for the solution of fluid model. Consider the nonlinear time fractional-order physical model of the form

$$
v_{t}^{\vartheta}(\Im, \Re, \xi, t)=\mathcal{L} v(\Im, \Re, \xi, t)+\aleph v(\Im, \Re, \xi, t)+g(\Im, \Re, \xi, t), \quad \vartheta \in[1,2],
$$

with initial source

$$
v(\Im, \Re, \xi, 0)=v(\Im, \Re, \xi),
$$

where $\mathcal{L}$ is a linear operator, whereas $\aleph$ is a nonlinear operator.

For computational procedure, we define some basic definitions and results.

Lemma 3.1 ([26]) For $v(\Im, \Re, \xi, t)=\sum_{0}^{\infty} \rho^{k} v_{k}(\Im, \Re, \xi, t)$, the linear operator $\mathcal{L} v(\Im, \Re, \xi, t)$ has the following property:

$$
\mathcal{L} v(\Im, \Re, \xi, t)=\mathcal{L}\left(\sum_{k=0}^{\infty} \rho^{k} v_{k}(\Im, \Re, \xi, t)\right)=\sum_{k=0}^{\infty} \mathcal{L}\left(\rho^{k} v_{k}(\Im, \Re, \xi, t)\right) .
$$

Theorem $3.2([26])$ Let $v(\Im, \Re, \xi, t)=\sum_{0}^{\infty} v_{k}(\Im, \Re, \xi, t)$. For the parameter $\lambda$, we define $v_{\lambda}(\Im, \Re, \xi, t)=\sum_{0}^{\infty} \lambda^{k} v_{k}(\Im, \Re, \xi, t)$. Then the nonlinear operator $\aleph v(\Im, \Re, \xi, t)$ satisfies the following property:

$$
\aleph\left(v_{\lambda}\right)=\aleph\left(\sum_{0}^{\infty} \lambda^{k} v_{k}(\Im, \Re, \xi, t)\right)=\sum_{0}^{\infty}\left[\frac{1}{n !} \frac{d^{n}}{d \lambda^{n}}\left[\aleph\left(\sum_{0}^{\infty} \lambda^{k} v_{k}(\Im, \Re, \xi, t)\right)\right]_{\lambda=0}\right] \lambda^{n} .
$$

Definition 3.3 ([1]) The polynomial $P_{n}=P_{n}\left(u_{0}, u_{1}, \ldots, u_{n}\right)$ is defined as

$$
P_{n}\left(u_{0}, u_{1}, \ldots, u_{n}\right)=\frac{1}{n !} \frac{d^{n}}{d \lambda^{n}}\left[\aleph\left(\sum_{0}^{\infty} \lambda^{k} v_{k}(\Im, \Re, \xi, t)\right)\right]_{\lambda=0} .
$$

Definition 3.4 ([26]) If $P_{n}=P_{n}\left(u_{0}, u_{1}, \ldots, u_{n}\right)$, then by definition (8) the nonlinear operator $\aleph\left(v_{\lambda}\right)$ is expressed as

$$
\aleph\left(v_{\lambda}\right)=\sum_{0}^{\infty} \lambda^{k} P_{k}
$$




\subsection{The existence and uniqueness of NAAM solution [26]}

The existence of NAAM is discussed by the following theorem.

Theorem 3.5 Let $g(\mathfrak{I}, \Re, \xi, t), v(\Im, \Re, \xi, t)$ be defined for $m-1<\vartheta<m$ in (5). Then the fluid model (5) provides the unique solution in the form

$$
v(\Im, \Re, \xi, t)=g_{t}^{-\vartheta}(\Im, \Re, \xi, t)+v(\Im, \Re, \xi)+\sum_{k=1}^{\infty}\left[\mathcal{L}_{t}^{-\vartheta}\left(v_{(k-1)}\right)+P_{(k-1) t}^{-\vartheta}\right]
$$

where, $\mathcal{L}_{t}^{-\vartheta}\left(v_{(k-1)}\right)$ and $P_{(k-1) t}^{-\vartheta}$ represent the fractional partial integrals of order $\vartheta$ for $\mathcal{L}\left(v_{k-1}\right)$ and $P_{(k-1)}$ with respect to $t$.

Proof Consider the solution of physical model $v(\Im, \Re, \xi, t)$ obtained by using the following expansion:

$$
v(\Im, \Re, \xi, t)=\sum_{k=0}^{\infty} v_{k}(\Im, \Re, \xi, t) .
$$

To solve the physical model with initial source (5), we consider

$$
v_{t \lambda}^{\vartheta}(\Im, \Re, \xi, t)=\lambda[\mathcal{L} v(\Im, \Re, \xi, t)+\aleph v(\Im, \Re, \xi, t)+g(\Im, \Re, \xi, t)], \quad \lambda \in[0,1],
$$

along with initial source

$$
v(\Im, \Re, \xi, 0)=v(\Im, \Re, \xi) .
$$

Furthermore, we approximate the solution of equation (10) as

$$
v_{\lambda}(\Im, \Re, \xi, t)=\sum_{0}^{\infty} \lambda^{k} v_{\lambda}(\Im, \Re, \xi, t) .
$$

Using the Riemann-Liouville fractional partial integral operator of order $\vartheta$ with respect to $t$ on both sides of equation (12) and thus using the properties of the Riemann-Liouuille fractional order operator (4), we obtain

$$
v_{\lambda}(\Im, \Re, \xi, t)=v(\Im, \Re, \xi, 0)+\lambda I_{t}^{\vartheta}[\mathcal{L} v(\Im, \Re, \xi, t)+\aleph v(\Im, \Re, \xi, t)+g(\Im, \Re, \xi, t)]
$$

By using the initial source (13) equation (15) becomes

$$
v_{\lambda}(\Im, \Re, \xi, t)=v(\Im, \Re, \xi)+\lambda I_{t}^{\vartheta}[\mathcal{L} v(\Im, \Re, \xi, t)+\aleph v(\Im, \Re, \xi, t)+g(\Im, \Re, \xi, t)] .
$$

Substituting equation (14) into equation (16), we have

$$
\begin{aligned}
\sum_{k=0}^{\infty} \lambda^{k} v_{\lambda}(\Im, \Re, \xi, t)= & v(\Im, \Re, \xi)+\lambda[g(\Im, \Re, \xi, t)] \\
& +\lambda I_{t}^{\vartheta}\left[\mathcal{L}\left(\sum_{k=0}^{\infty} \lambda^{k} v(\Im, \Re, \xi, t)\right)+\aleph\left(\sum_{k=0}^{\infty} \lambda^{k} v(\Im, \Re, \xi, t)\right)\right] .
\end{aligned}
$$


Using Lemma 3.1 and Definition 3.4, equation (17) becomes

$$
\begin{aligned}
\sum_{k=0}^{\infty} \lambda^{k} v_{\lambda}(\Im, \Re, \xi, t)= & v(\Im, \Re, \xi)+\lambda[g(\Im, \Re, \xi, t)] \\
& +\lambda I_{t}^{\vartheta}\left[\mathcal{L}\left(\sum_{k=0}^{\infty} \lambda^{k} \lambda^{k} v_{k}\right)\right]+\lambda I_{t}^{\vartheta}\left[\aleph\left(\sum_{k=0}^{\infty} \lambda^{k} P_{n}\right)\right]
\end{aligned}
$$

Equating the coefficients at the same powers of $\lambda$ in equation (18), we obtain the component form

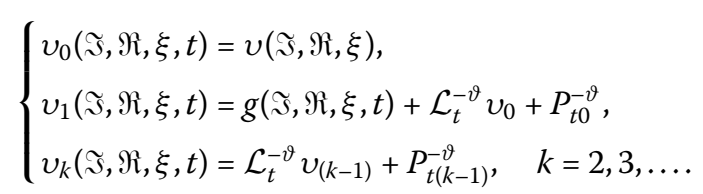

\section{NAAM implementation and discussion of results [26]}

In this section, we use NAAM for analytical analysis of some mathematical models related to fluid mechanics.

Example 4.1 Consider the time fractional-order Naiver-Stokes model along with initial source in the form [22];

$$
\frac{\partial^{\vartheta} v(\mathfrak{\Im}, t)}{\partial t^{\vartheta}}=\xi+\frac{\partial^{2} v(\mathfrak{I}, t)}{\partial \mathfrak{I}^{2}}+\frac{1}{\mathfrak{I}}\left(\frac{\partial v(\mathfrak{\Im}, t)}{\partial \mathfrak{I}}\right), \quad \vartheta \in(0,1]
$$

with initial source

$$
v(\mathfrak{s}, 0)=1-\mathfrak{\Im}^{2}
$$

where $\xi=-\frac{1}{\rho} \frac{\partial p}{\partial z}=1$. In the particular case $\vartheta=1$ the exact solution is

$$
u(\mathfrak{s}, t)=1-\mathfrak{\Im}^{2}-3 t
$$

To solve the physical model (20), comparing it with equation (5), we get

$$
\frac{\partial^{\vartheta} v(\mathfrak{I}, t)}{\partial t^{\vartheta}}=\xi+\frac{\partial^{2} v(\Im, t)}{\partial \Im^{2}}+\aleph(v(\mathfrak{\Im}, t)), \quad \vartheta \in(0,1],
$$

where the nonlinear term $\aleph(v(\mathfrak{I}, t))=\frac{1}{\Im}\left(\frac{\partial v(\Im, t)}{\partial \Im}\right)$.

The approximate solution of equation (20) is assumed as

$$
v(\mathfrak{I}, t)=\sum_{k=0}^{\infty} v_{k}(\mathfrak{I}, t)
$$

For obtaining the analytical solution of equation (21), we use the procedure

$$
\frac{\partial^{\vartheta} v(\Im, t)}{\partial t^{\vartheta}}=\lambda\left[\xi+\frac{\partial^{2} v(\Im, t)}{\partial \Im^{2}}+\aleph(v(\mathfrak{I}, t))\right], \quad \vartheta \in(0,1]
$$


with initial source

$$
v(\mathfrak{I}, 0)=1-\mathfrak{\Im}^{2} .
$$

We assume that equation (23) has the solution of the form

$$
v_{\lambda}(\mathfrak{I}, t)=\sum_{k=0}^{\infty} \lambda^{k} v_{k}(\Im, t)
$$

Now by using Riemann-Liouville fractional-order integration with respect to $t$ on both sides of equation (23), its properties (2), and initial source (24), we obtain

$$
v_{\lambda}(\mathfrak{\Im}, t)=v(\mathfrak{\Im}, 0)+\lambda I_{t}^{\vartheta}\left[\xi+\frac{\partial^{2} v(\mathfrak{s}, t)}{\partial \mathfrak{\Im}^{2}}+\aleph(v(\mathfrak{I}, t))\right]
$$

Use equation (25), we obtain

$$
\sum_{k=0}^{\infty} \lambda^{k} v_{k}(\Im, t)=v(\Im, 0)+\lambda I_{t}^{\vartheta}\left[\xi+\sum_{k=0}^{\infty} \lambda^{k}\left(\frac{\partial^{2} v_{k}(\Im, t)}{\partial \Im^{2}}\right)+\sum_{k=0}^{\infty} \lambda^{k} P_{k}\right]
$$

Equating the terms with identical powers of $\lambda$ of equation (27), we get the final components and recursive relation of the form

$$
\left\{\begin{array}{l}
v_{0}(\Im, t)=v(\Im, 0) \\
v_{1}(\Im, t)=I_{t}^{\vartheta}\left[\xi+\left(\frac{\partial^{2} v_{0}(\Im, t)}{\partial \Im^{2}}\right)+P_{0}\right] \\
v_{k}(\Im, \Re, \xi, t)=I_{t}^{\vartheta}\left[\xi+\left(\frac{\partial^{2} v_{(k-1)}(\Im, t)}{\partial \Im^{2}}\right)+P_{(k-1)}\right]
\end{array}\right.
$$

Thus we obtain

$$
\begin{aligned}
& v_{0}(\Im, t)=1-\Im^{2}, \\
& v_{1}(\Im, t)=(\xi-4) \frac{t^{\vartheta}}{\Gamma(\vartheta+1)}, \\
& v_{2}(\Im, t)=0, \\
& v_{3}(\Im, t)=0 .
\end{aligned}
$$

The NAAM solution is

$$
v(\mathfrak{I}, t)=v_{0}(\mathfrak{I}, t)+v_{1}(\mathfrak{\Im}, t)+v_{2}(\mathfrak{\Im}, t)+v_{3}(\mathfrak{I}, t)+\cdots
$$

By putting the corresponding obtained values we get

$$
v(\Im, t)=1-\Im^{2}+(\xi-4) \frac{t^{\vartheta}}{\Gamma(\vartheta+1)} .
$$


Figure 1 The exact and NAAM solution graphs of Example 4.1

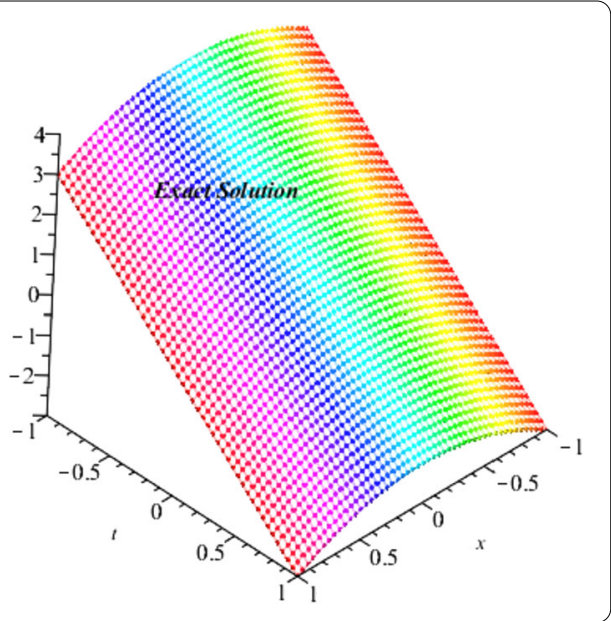

Figure 2 The fractional-order graph of Example 4.1

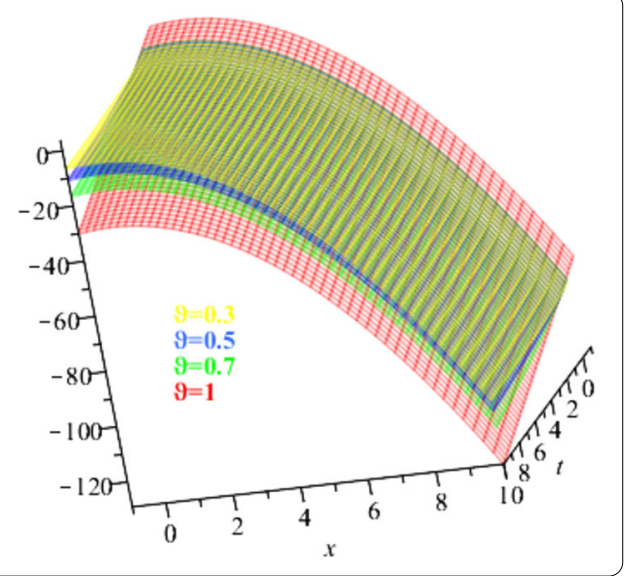

Specifically, for $\vartheta=\xi=1$, the solution becomes the given exact solution

$$
v(\Im, t)=1-\Im^{2}-3 t .
$$

Example 4.2 The time fractional Navier-Stokes model is given as [22]

$$
\frac{\partial^{\vartheta} v(\Im, t)}{\partial t^{\vartheta}}=\frac{\partial^{2} v(\Im, t)}{\partial \Im^{2}}+\frac{1}{\Im}\left(\frac{\partial v(\Im, t)}{\partial \Im}\right), \quad \vartheta \in(0,1]
$$

with initial source

$$
v(\mathfrak{I}, 0)=\mathfrak{I},
$$

where $\xi=-\frac{1}{\rho} \frac{\partial p}{\partial z}=1$. In the particular case $\vartheta=1$, the exact solution is

$$
u(\Im, t)=\Im+\sum_{m=1}^{\infty} \frac{1^{2} \times 3^{2} \cdots(2 m-3)^{2}}{\Im^{2 m-1}} \cdot \frac{t^{m}}{\vartheta !} .
$$


Comparing equations (36) and (5), we have

$$
\frac{\partial^{\vartheta} v(\mathfrak{\Im}, t)}{\partial t^{\vartheta}}=\frac{\partial^{2} v(\mathfrak{\Im}, t)}{\partial \mathfrak{\Im}^{2}}+\aleph(v(\mathfrak{I}, t)), \quad \vartheta \in(0,1]
$$

where the nonlinear term $\aleph(v(\Im, t))=\frac{1}{\Im}\left(\frac{\partial v(\Im, t)}{\partial \mathfrak{I}}\right)$.

The approximate solution of equation (36) is assumed as

$$
v(\mathfrak{s}, t)=\sum_{k=0}^{\infty} v_{k}(\mathfrak{s}, t)
$$

We obtain the solution of equation (36) by using the following procedure:

$$
\frac{\partial^{\vartheta} v(\Im, t)}{\partial t^{\vartheta}}=\lambda\left[\frac{\partial^{2} v(\Im, t)}{\partial \Im^{2}}+\aleph(v(\Im, t))\right], \quad \vartheta \in(0,1],
$$

with initial source

$$
v(\mathfrak{I}, 0)=\mathfrak{\Im} .
$$

We assume that equation (38) has the solution of the form

$$
v_{\lambda}(\Im, t)=\sum_{k=0}^{\infty} \lambda^{k} v_{k}(\Im, t)
$$

Using Riemann-Liouville fractional-order integration with respect to $t$ of both sides of equation (39), its properties (2), and initial source (40), we obtain

$$
v_{\lambda}(\mathfrak{\Im}, t)=v(\mathfrak{I}, 0)+\lambda I_{t}^{\vartheta}\left[\frac{\partial^{2} v(\mathfrak{I}, t)}{\partial \mathfrak{\Im}^{2}}+\aleph(v(\mathfrak{I}, t))\right] .
$$

Using definition (9) and equation (41), we obtain

$$
\sum_{k=0}^{\infty} \lambda^{k} v_{k}(\mathfrak{I}, t)=v(\mathfrak{I}, 0)+\lambda I_{t}^{\vartheta}\left[\sum_{k=0}^{\infty} \lambda^{k}\left(\frac{\partial^{2} v_{k}(\mathfrak{I}, t)}{\partial \mathfrak{I}^{2}}\right)+\sum_{k=0}^{\infty} \lambda^{k} P_{k}\right]
$$

Equating the terms with identical powers of $\lambda$ of equation (27), we get the following recursive scheme:

$$
\left\{\begin{array}{l}
v_{0}(\Im, t)=v(\Im, 0), \\
v_{1}(\Im, t)=I_{t}^{\vartheta}\left[\left(\frac{\partial^{2} v_{0}(\Im, t)}{\partial \Im^{2}}\right)+P_{0}\right], \\
v_{k}(\Im, \Re, \xi, t)=I_{t}^{\vartheta}\left[\rho+\left(\frac{\partial^{2} v_{(k-1)}(\Im, t)}{\partial \Im^{2}}\right)+P_{(k-1)}\right] .
\end{array}\right.
$$

Thus we obtain

$$
\begin{aligned}
& v_{0}(\mathfrak{\Im}, t)=\mathfrak{\Im} \\
& v_{1}(\mathfrak{s}, t)=\left(\frac{1}{\mathfrak{I}}\right) \frac{t^{\vartheta}}{\Gamma(\vartheta+1)},
\end{aligned}
$$




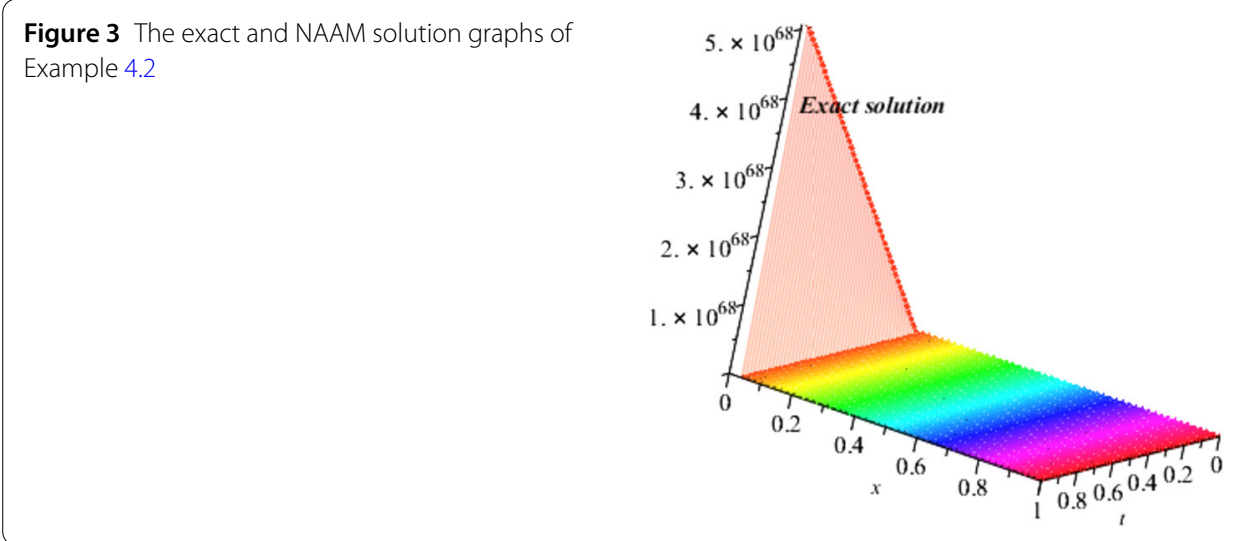

Figure 4 The fractional-order graph of Example 4.2

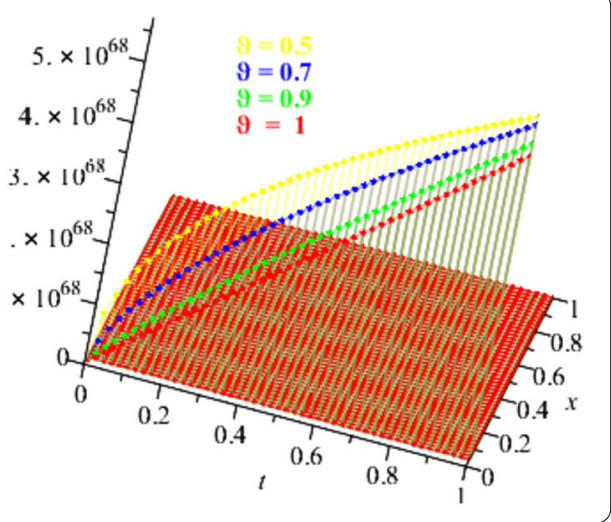

$$
\begin{aligned}
& v_{2}(\mathfrak{\Im}, t)=\left(\frac{1}{\mathfrak{\Im}^{3}}\right) \frac{t^{2 \vartheta}}{\Gamma(2 \vartheta+1)}, \\
& v_{3}(\mathfrak{I}, t)=\left(\frac{9}{\mathfrak{\Im}^{5}}\right) \frac{t^{3 \vartheta}}{\Gamma(3 \vartheta+1)} .
\end{aligned}
$$

The NAAM solution is

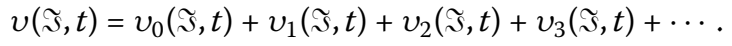

Using the NAAM components, we have

$$
v(\mathfrak{I}, t)=\mathfrak{\Im}+\left(\frac{1}{\mathfrak{s}}\right) \frac{t^{\vartheta}}{\Gamma(\vartheta+1)}+\left(\frac{1}{\mathfrak{\Im}^{3}}\right) \frac{t^{2 \vartheta}}{\Gamma(2 \vartheta+1)}+\left(\frac{9}{\mathfrak{\Im}^{5}}\right) \frac{t^{3 \vartheta}}{\Gamma(3 \vartheta+1)}
$$

Specifically, for $\vartheta=\rho=1$, the solution becomes the given exact solution

$$
v(\Im, t)=\Im+\sum_{m=1}^{\infty} \frac{1^{2} \times 3^{2} \cdots(2 m-3)^{2}}{\Im^{2 m-1}} \cdot \frac{t^{m}}{\vartheta !} .
$$


Example 4.3 Consider the time fractional-order incompressible models of NavierStokes with initial source in the form $[25,26]$

$$
\left\{\begin{array}{l}
\frac{\partial^{\vartheta} u}{\partial t^{\vartheta}}+u \frac{\partial u}{\partial \Im}+v \frac{\partial u}{\partial \Re}=\rho_{0}\left(\frac{\partial^{2} u}{\partial \Im^{2}}+\frac{\partial^{2} u}{\partial \Re^{2}}\right)+g, \\
\frac{\partial^{\vartheta} v}{\partial t^{\vartheta}}+u \frac{\partial v}{\partial \Im}+v \frac{\partial v}{\partial \Re}=\rho_{0}\left(\frac{\partial^{2} v}{\partial \Im^{2}}+\frac{\partial^{2} v}{\partial \Re^{2}}\right)-g,
\end{array} \quad \vartheta \in(0,1]\right.
$$

with initial sources

$$
\begin{aligned}
& u(\Im, \Re, 0)=-e^{\Im+\Re}, \\
& v(\Im, \Re, 0)=e^{\Im+\Re} .
\end{aligned}
$$

We assume that equation (52) has the solution of the form

$$
v_{\lambda}(\Im, t)=\sum_{k=0}^{\infty} \lambda^{k} v_{k}(\Im, t) .
$$

Using Riemann-Liouville fractional order integration with respect to $t$ on both sides of equation (52), its properties (2), and initial sources, we obtain

$$
\begin{aligned}
& u_{\lambda}(\Im, t)=u(\Im, 0)+\lambda I_{t}^{\vartheta}\left[-u \frac{\partial u}{\partial \Im}-v \frac{\partial u}{\partial \Re}+\rho_{0}\left(\frac{\partial^{2} u}{\partial \Im^{2}}+\frac{\partial^{2} u}{\partial \Re^{2}}\right)+g\right], \\
& v_{\lambda}(\Im, t)=v(\Im, 0)+\lambda I_{t}^{\vartheta}\left[-u \frac{\partial v}{\partial \Im}-v \frac{\partial v}{\partial \Re}+\rho_{0}\left(\frac{\partial^{2} v}{\partial \Im^{2}}+\frac{\partial^{2} v}{\partial \Re^{2}}\right)-g\right] .
\end{aligned}
$$

By (9) and equation (54) we obtain

$$
\begin{aligned}
& \sum_{k=0}^{\infty} \lambda^{k} u_{k}(\Im, t)=u(\Im, 0)+\lambda I_{t}^{\vartheta}\left[-\sum_{k=0}^{\infty} \lambda^{k} P_{k}-\sum_{k=0}^{\infty} \lambda^{k} q_{k}+\rho_{0}\left(\frac{\partial^{2} u}{\partial \Im^{2}}+\frac{\partial^{2} u}{\partial \Re^{2}}\right)+g\right], \\
& \sum_{k=0}^{\infty} \lambda^{k} v_{k}(\Im, t)=v(\Im, 0)+\lambda I_{t}^{\vartheta}\left[-\sum_{k=0}^{\infty} \lambda^{k} P_{k}^{*}-\sum_{k=0}^{\infty} \lambda^{k} q_{k}^{*}+\rho_{0}\left(\frac{\partial^{2} v}{\partial \Im^{2}}+\frac{\partial^{2} v}{\partial \Re^{2}}\right)-g\right] .
\end{aligned}
$$

Equating the terms with identical powers of $\lambda$ of equation (55), we get the final components of the system in the form of recursive relations

$$
\begin{aligned}
& \left\{\begin{array}{l}
u_{0}(\Im, \Re, t)=u(\Im, \Re, 0) \\
u_{1}(\Im, t)=\lambda I_{t}^{\vartheta}\left[-P_{0}-q_{0}+\rho_{0}\left(\frac{\partial^{2} u_{0}}{\partial \Im^{2}}+\frac{\partial^{2} u_{0}}{\partial \Re^{2}}\right)+g\right], \\
u_{k}(\Im, \Re, \xi, t)=\lambda I_{t}^{\vartheta}\left[-P_{k-1}-q_{k-1}+\rho_{0}\left(\frac{\partial^{2} u_{k-1}}{\partial \Im^{2}}+\frac{\partial^{2} u_{k-1}}{\partial \Re^{2}}\right)+g\right],
\end{array}\right. \\
& \left\{\begin{array}{l}
v_{0}(\Im, \Re, t)=u(\Im, \Re, 0) \\
v_{1}(\Im, t)=\lambda I_{t}^{\vartheta}\left[-P_{0}^{*}-q_{0}^{*}+\rho_{0}\left(\frac{\partial^{2} u_{0}}{\partial \Im^{2}}+\frac{\partial^{2} u_{0}}{\partial \Re^{2}}\right)-g\right], \\
v_{k}(\Im, \Re, \xi, t)=\lambda I_{t}^{\vartheta}\left[-P_{k-1}^{*}-q_{k-1}^{*}+\rho_{0}\left(\frac{\partial^{2} v_{k-1}}{\partial \Im^{2}}+\frac{\partial^{2} v_{k-1}}{\partial \Re^{2}}\right)-g\right] .
\end{array}\right.
\end{aligned}
$$

Thus we obtain the terms of NAAM of equation (56) as

$$
u_{0}(\Im, \Re, t)=u(\Im, \Re, 0)=-e^{\Im+\Re},
$$




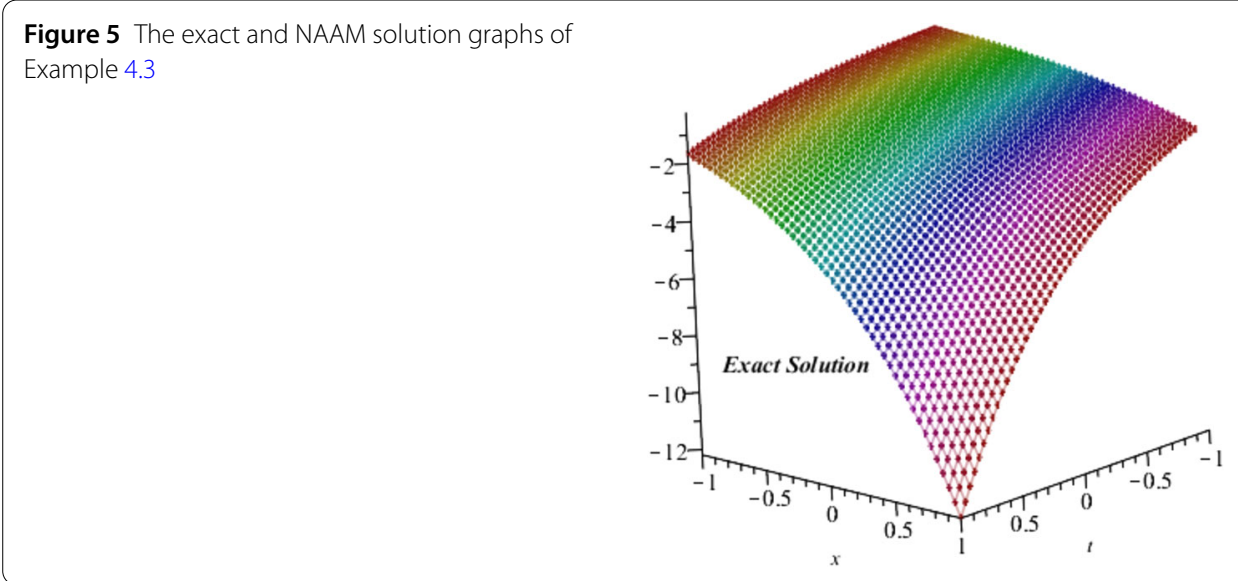

$$
\begin{aligned}
& u_{1}(\Im, \Re, t)=2 \rho_{0}\left(-e^{\Im+\Re}\right) \frac{t^{\vartheta}}{\Gamma(\vartheta+1)}, \\
& u_{2}(\Im, \Re, t)=4 \rho_{0}^{2}\left(-e^{\Im+\Re}\right) \frac{t^{2 \vartheta}}{\Gamma(2 \vartheta+1)}, \\
& u_{3}(\Im, \Re, t)=8 \rho_{0}^{3}\left(-e^{\Im+\Re}\right) \frac{t^{3 \vartheta}}{\Gamma(3 \vartheta+1)},
\end{aligned}
$$

Thus we also obtain the terms of NAAM of equation (57) as

$$
\begin{aligned}
& v_{0}(\Im, \Re, t)=v(\Im, \Re, 0)=e^{\Im+\Re}, \\
& v_{1}(\Im, \Re, t)=2 \rho_{0}\left(e^{\Im+\Re}\right) \frac{t^{\vartheta}}{\Gamma(\vartheta+1)}, \\
& v_{2}(\Im, \Re, t)=4 \rho_{0}^{2}\left(e^{\Im+\Re}\right) \frac{t^{2} \vartheta}{\Gamma(2 \vartheta+1)}, \\
& v_{3}(\Im, \Re, t)=8 \rho_{0}^{3}\left(e^{\Im+\Re}\right) \frac{t^{3} \vartheta}{\Gamma(3 \vartheta+1)},
\end{aligned}
$$

Thus the NAAM solution becomes

$$
\left\{\begin{array}{l}
u=u_{0}+u_{1}+u_{2}+u_{3}+\cdots \\
v=v_{0}+v_{1}+v_{2}+v_{3}+\cdots
\end{array}\right.
$$

Putting the corresponding values of $u$ and $v$ into (58), (59), we get

$$
\left\{\begin{aligned}
u(\Im, \Re, t)= & -e^{\Im+\Re}+2 \rho_{0}\left(-e^{\Im+\Re}\right) \frac{t^{\vartheta}}{\Gamma(\vartheta+1)}+4 \rho_{0}^{2}\left(-e^{\Im+\Re}\right) \frac{t^{2 \vartheta}}{\Gamma(2 \vartheta+1)} \\
& +8 \rho_{0}^{3}\left(-e^{\Im+\Re}\right) \frac{t^{3 \vartheta}}{\Gamma(3 \vartheta+1)}+\cdots \\
v(\Im, \Re, t)= & e^{\Im+\Re}+2 \rho_{0}\left(e^{\Im+\Re}\right) \frac{t^{\vartheta}}{\Gamma(\vartheta+1)}+4 \rho_{0}^{2}\left(e^{\Im+\Re}\right) \frac{t^{2 \vartheta}}{\Gamma(2 \vartheta+1)} \\
& +8 \rho_{0}^{3}\left(e^{\Im+\Re}\right) \frac{t^{3 \vartheta}}{\Gamma(3 \vartheta+1)}+\cdots
\end{aligned}\right.
$$




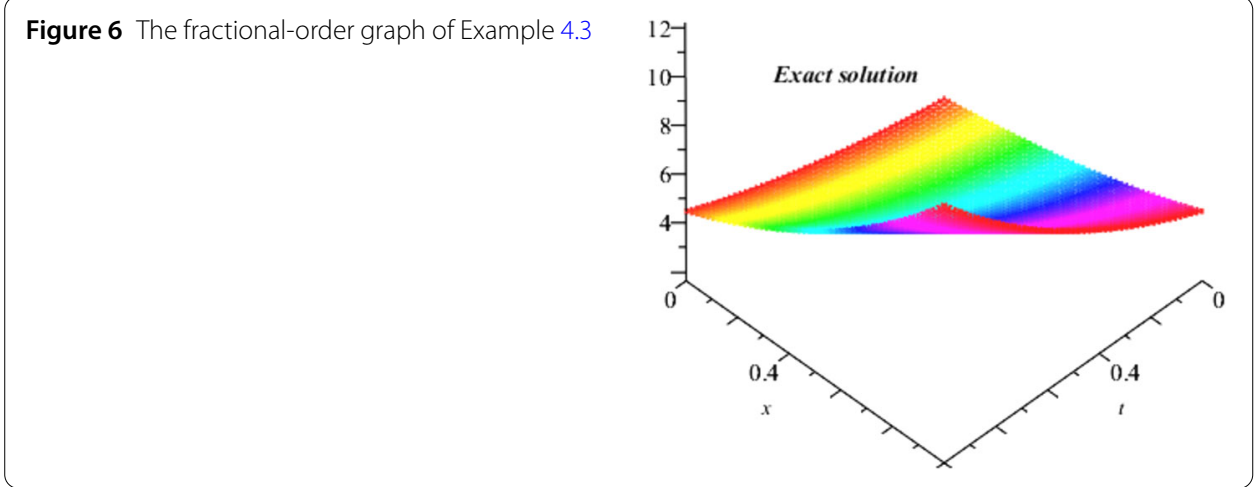

Figure 7 The different fractional-order graph of Example 4.3

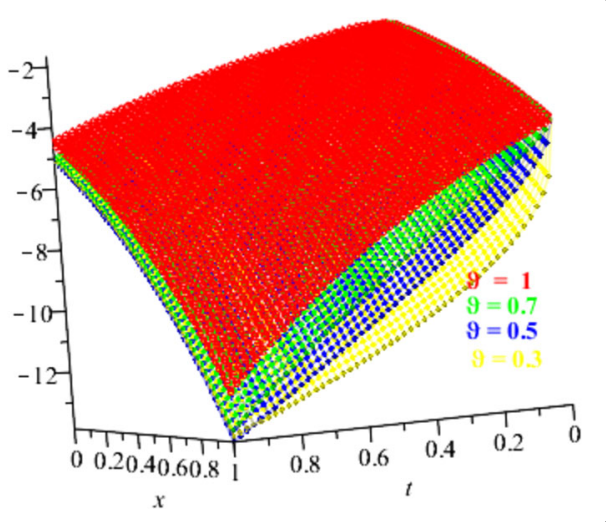

Figure 8 The different fractional-order graph of Example 4.3

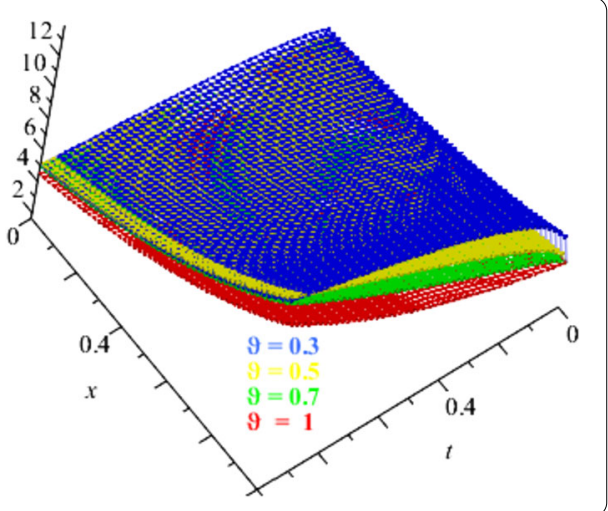

For the particular case of integer order $\vartheta=1$, we convert the NAAM result to the exact form solution

$$
\left\{\begin{array}{l}
u(\Im, \Re, t)=-e^{\left(\Im+\Re+2 \rho_{0} t\right)} \\
v(\Im, \Re, t)=e^{\left(\Im+\Re+2 \rho_{0} t\right)}
\end{array}\right.
$$

Example 4.4 Consider the time fractional-order incompressible models of NavierStokes with initial source in the form $[25,26]$

$$
\left\{\begin{array}{l}
\frac{\partial^{\vartheta} u}{\partial t^{\vartheta}}+u \frac{\partial u}{\partial \Im}+v \frac{\partial u}{\partial \Re}=\rho_{0}\left(\frac{\partial^{2} u}{\partial \Im^{2}}+\frac{\partial^{2} u}{\partial \Re^{2}}\right)+g, \\
\frac{\partial^{\vartheta} v}{\partial t^{\vartheta}}+u \frac{\partial v}{\partial \Im}+v \frac{\partial v}{\partial \Re}=\rho_{0}\left(\frac{\partial^{2} v}{\partial \Im^{2}}+\frac{\partial^{2} v}{\partial \Re^{2}}\right)-g,
\end{array} \quad \vartheta \in(0,1]\right.
$$


with initial sources

$$
\begin{aligned}
& u(\Im, \Re, 0)=-\sin (\Im+\Re), \\
& v(\Im, \Re, 0)=\sin (\Im+\Re) .
\end{aligned}
$$

We assume that equation (63) has the solution of the form

$$
v_{\lambda}(\Im, \Re, t)=\sum_{k=0}^{\infty} \lambda^{k} v_{k}(\Im, \Re, t) .
$$

Now by using the Riemann-Liouville operator with respect to $t$ on both sides of equation (63), its properties (2), and initial sources, we obtain

$$
\begin{aligned}
& u_{\lambda}(\Im, \Re, t)=u(\Im, \Re, 0)+\lambda I_{t}^{\vartheta}\left[-u \frac{\partial u}{\partial \Im}-v \frac{\partial u}{\partial \Re}+\rho_{0}\left(\frac{\partial^{2} u}{\partial \Im^{2}}+\frac{\partial^{2} u}{\partial \Re^{2}}\right)+g\right] \\
& v_{\lambda}(\Im, \Re, t)=v(\Im, \Re, 0)+\lambda I_{t}^{\vartheta}\left[-u \frac{\partial v}{\partial \Im}-v \frac{\partial v}{\partial \Re}+\rho_{0}\left(\frac{\partial^{2} v}{\partial \Im^{2}}+\frac{\partial^{2} v}{\partial \Re^{2}}\right)-g\right] .
\end{aligned}
$$

Use definition (9) and equation (65), we obtain

$$
\begin{aligned}
\sum_{k=0}^{\infty} \lambda^{k} u_{k}(\Im, \Re, t)= & u(\Im, \Re, 0) \\
& +\lambda I_{t}^{\vartheta}\left[-\sum_{k=0}^{\infty} \lambda^{k} P_{k}-\sum_{k=0}^{\infty} \lambda^{k} q_{k}+\rho_{0}\left(\frac{\partial^{2} u}{\partial \Im^{2}}+\frac{\partial^{2} u}{\partial \Re^{2}}\right)+g\right], \\
\sum_{k=0}^{\infty} \lambda^{k} v_{k}(\Im, \Re, t)= & v(\Im, \Re, 0) \\
& +\lambda I_{t}^{\vartheta}\left[-\sum_{k=0}^{\infty} \lambda^{k} P_{k}^{*}-\sum_{k=0}^{\infty} \lambda^{k} q_{k}^{*}+\rho_{0}\left(\frac{\partial^{2} v}{\partial \Im^{2}}+\frac{\partial^{2} v}{\partial \Re^{2}}\right)-g\right] .
\end{aligned}
$$

Equating the terms with identical powers of $\lambda$ of equation (66), we get the final components and recursive relation in the form

$$
\begin{aligned}
& \left\{\begin{array}{l}
u_{0}(\Im, \Re, t)=u(\Im, \Re, 0), \\
u_{1}(\Im, t)=\lambda I_{t}^{\vartheta}\left[-P_{0}-q_{0}+\rho_{0}\left(\frac{\partial^{2} u_{0}}{\partial \Im^{2}}+\frac{\partial^{2} u_{0}}{\partial \Re^{2}}\right)+g\right], \\
u_{k}(\Im, \Re, \xi, t)=\lambda I_{t}^{\vartheta}\left[-P_{k-1}-q_{k-1}+\rho_{0}\left(\frac{\partial^{2} u_{k-1}}{\partial \Im^{2}}+\frac{\partial^{2} u_{k-1}}{\partial \Re^{2}}\right)+g\right],
\end{array}\right. \\
& \left\{\begin{array}{l}
v_{0}(\Im, \Re, t)=u(\Im, \Re, 0), \\
v_{1}(\Im, t)=\lambda I_{t}^{\vartheta}\left[-P_{0}^{*}-q_{0}^{*}+\rho_{0}\left(\frac{\partial^{2} u_{0}}{\partial \Im^{2}}+\frac{\partial^{2} u_{0}}{\partial \Re^{2}}\right)+g\right], \\
v_{k}(\Im, \Re, \xi, t)=\lambda I_{t}^{\vartheta}\left[-P_{k-1}^{*}-q_{k-1}^{*}+\rho_{0}\left(\frac{\partial^{2} v_{k-1}}{\partial \Im^{2}}+\frac{\partial^{2} v_{k-1}}{\partial \Re^{2}}\right)+g\right] .
\end{array}\right.
\end{aligned}
$$

Thus we obtain the NAAM term of equation (67) in the form

$$
\begin{aligned}
& u_{0}(\Im, \Re, t)=-\sin (\Im+\Re), \\
& u_{1}(\Im, \Re, t)=2 \rho_{0}(\sin (\Im+\Re)) \frac{t^{\vartheta}}{\Gamma(\vartheta+1)},
\end{aligned}
$$




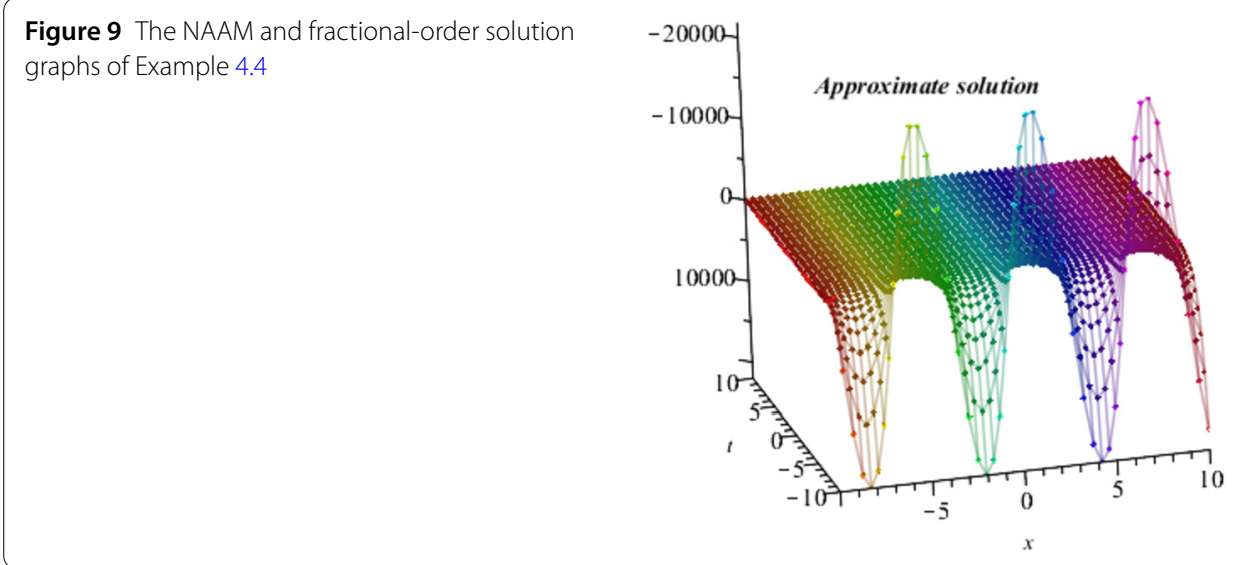

Figure 10 The different fractional-order graph of Example 4.4

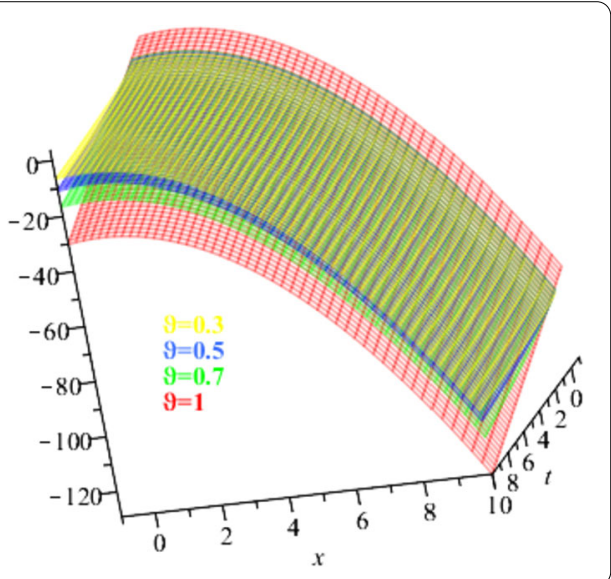

Figure 11 The NAAM and fractional-order solution graphs of Example 4.4

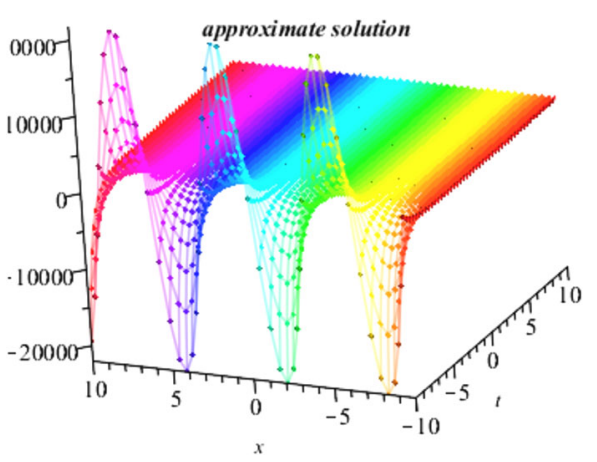

$$
\begin{aligned}
& u_{2}(\Im, \Re, t)=-4 \rho_{0}(\sin (\Im+\Re)) \frac{t^{2 \vartheta}}{\Gamma(2 \vartheta+1)}, \\
& u_{3}(\Im, \Re, t)=8 \rho_{0}(\sin (\Im+\Re)) \frac{t^{3 \vartheta}}{\Gamma(3 \vartheta+1)},
\end{aligned}
$$

Thus we can also obtain the NAAM term of equation (68) in the form 
Figure 12 The fractional-order graph of Example 4.4

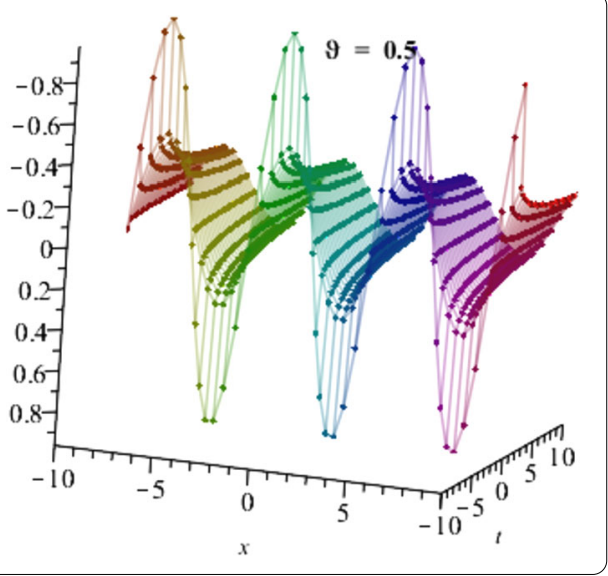

$$
\begin{aligned}
& v_{0}(\Im, \Re, t)=\sin (\Im+\Re), \\
& v_{1}(\Im, \Re, t)=-2 \rho_{0}(\sin (\Im+\Re)) \frac{t^{\vartheta}}{\Gamma(\vartheta+1)}, \\
& v_{2}(\Im, \Re, t)=4 \rho_{0}(\sin (\Im+\Re)) \frac{t^{2 \vartheta}}{\Gamma(2 \vartheta+1)}, \\
& v_{3}(\Im, \Re, t)=-8 \rho_{0}(\sin (\Im+\Re)) \frac{t^{3 \vartheta}}{\Gamma(3 \vartheta+1)} .
\end{aligned}
$$

Thus the NAAM solution becomes

$$
\left\{\begin{array}{l}
u=u_{0}+u_{1}+u_{2}+u_{3}+\cdots \\
v=v_{0}+v_{1}+v_{2}+v_{3}+\cdots
\end{array}\right.
$$

By putting the NAAM components given into equations (69) and (70) we get

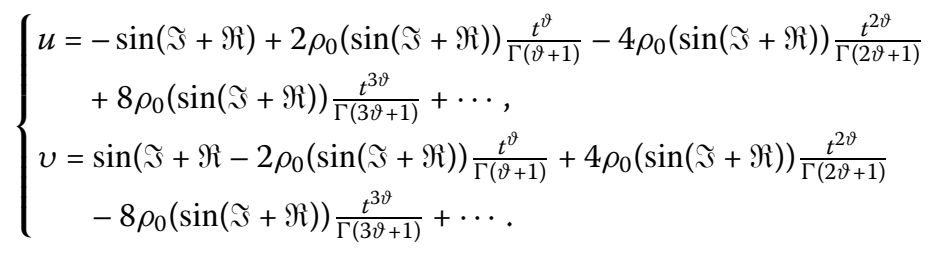

For the particular case of integer order $\vartheta=1$, the NAAM provides the exact form solution

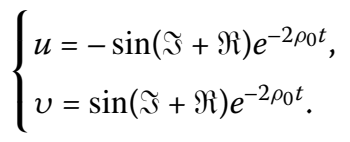


Example 4.5 Consider the three-dimensional time fractional-order incompressible models of Navier-Stokes with initial source in the form [25, 26]

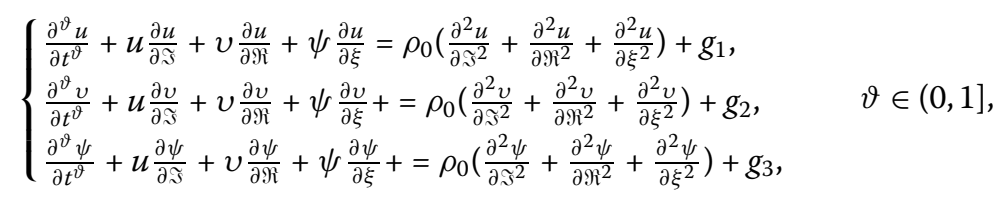

with initial sources

$$
\begin{aligned}
& u(\Im, \Re, \xi, 0)=-0.5 \Im+\Re+\xi \\
& v(\Im, \Re, \xi, 0)=\Im-0.5 \Re+\xi \\
& \psi(\Im, \Re, \xi, 0)=\Im+\Re-0.5 \xi,
\end{aligned}
$$

where $g_{1}=-\frac{1}{\rho} \frac{\partial p}{\partial \mathfrak{\Im}}, g_{2}=-\frac{1}{\rho} \frac{\partial p}{\partial \Re}, g_{3}=-\frac{1}{\rho} \frac{\partial p}{\partial \xi}$.

We assume that equation (74) has the solution in the form

$$
v_{\lambda}(\Im, \Re, t)=\sum_{k=0}^{\infty} \lambda^{k} v_{k}(\Im, \Re, t) .
$$

Using the Caputo operator with respect to $t$ on both sides of equation (74), its properties (2), and initial sources, we obtain

$$
\begin{aligned}
u_{\lambda}(\Im, \Re, \xi, t)= & u(\Im, \Re, \xi, 0) \\
& +\lambda I_{t}^{\vartheta}\left[-u \frac{\partial u}{\partial \Im}-v \frac{\partial u}{\partial \Re}-\psi \frac{\partial u}{\partial \xi}+\rho_{0}\left(\frac{\partial^{2} u}{\partial \Im^{2}}+\frac{\partial^{2} u}{\partial \Re^{2}}+\frac{\partial^{2} u}{\partial \xi^{2}}\right)+g_{1}\right], \\
v_{\lambda}(\Im, \Re, \xi, t)= & v(\Im, \Re, \xi, 0) \\
& +\lambda I_{t}^{\vartheta}\left[-u \frac{\partial v}{\partial \Im}-v \frac{\partial v}{\partial \Re}-\psi \frac{\partial v}{\partial \xi}+\rho_{0}\left(\frac{\partial^{2} v}{\partial \Im^{2}}+\frac{\partial^{2} v}{\partial \Re^{2}}+\frac{\partial^{2} v}{\partial \xi^{2}}\right)+g_{2}\right], \\
\psi_{\lambda}(\Im, \Re, \xi, t)= & \psi(\Im, \Re, \xi, 0) \\
& +\lambda I_{t}^{\vartheta}\left[-u \frac{\partial \psi}{\partial \Im}-v \frac{\partial \psi}{\partial \Re}-\psi \frac{\partial \psi}{\partial \xi}+\rho_{0}\left(\frac{\partial^{2} \psi}{\partial \Im^{2}}+\frac{\partial^{2} \psi}{\partial \Re^{2}}+\frac{\partial^{2} \psi}{\partial \xi^{2}}\right)+g_{3}\right] .
\end{aligned}
$$

Using definition (9) and equation (76), we obtain

$$
\begin{aligned}
& \sum_{k=0}^{\infty} \lambda^{k} u_{k}(\Im, \Re, \xi, t) \\
& =u(\Im, \Re, \xi, 0)+\lambda I_{t}^{\vartheta}\left[-\sum_{k=0}^{\infty} \lambda^{k} P_{k}-\sum_{k=0}^{\infty} \lambda^{k} q_{k}+\sum_{k=0}^{\infty} \lambda^{k} \delta_{k}\right. \\
& \left.\quad+\rho_{0}\left(\frac{\partial^{2} u}{\partial \Im^{2}}+\frac{\partial^{2} u}{\partial \Re^{2}}+\frac{\partial^{2} u}{\partial \xi^{2}}\right)+g_{1}\right],
\end{aligned}
$$




$$
\begin{aligned}
& \sum_{k=0}^{\infty} \lambda^{k} v_{k}(\Im, \Re, \xi, t) \\
& =v(\Im, \Re, \xi, 0)+\lambda I_{t}^{\vartheta}\left[-\sum_{k=0}^{\infty} \lambda^{k} P_{k}^{*}-\sum_{k=0}^{\infty} \lambda^{k} q_{k}^{*}+\sum_{k=0}^{\infty} \lambda^{k} \delta_{k}^{*}\right. \\
& \left.\quad+\rho_{0}\left(\frac{\partial^{2} v}{\partial \Im^{2}}+\frac{\partial^{2} v}{\partial \Re^{2}}+\frac{\partial^{2} v}{\partial \xi^{2}}\right)+g_{2}\right], \\
& \sum_{k=0}^{\infty} \lambda^{k} \psi_{k}(\Im, \Re, \xi, t) \\
& =\psi(\Im, \Re, \xi, 0)+\lambda I_{t}^{\vartheta}\left[-\sum_{k=0}^{\infty} \lambda^{k} P_{k}^{\bullet}-\sum_{k=0}^{\infty} \lambda^{k} q_{k}^{\bullet}+\sum_{k=0}^{\infty} \lambda^{k} \delta_{k}^{\bullet}\right. \\
& \left.\quad+\rho_{0}\left(\frac{\partial^{2} \psi}{\partial \Im^{2}}+\frac{\partial^{2} \psi}{\partial \Re^{2}}+\frac{\partial^{2} \psi}{\partial \xi^{2}}\right)+g_{3}\right],
\end{aligned}
$$

where, $p, q, \delta, p^{*}, q^{*}, \delta^{*}, p^{\bullet}, q^{\bullet}, \delta^{\bullet}$ represent the nonlinear terms of system (76).

Equating the terms with identical powers of $\lambda$ of equation (77), we get the final components and recursive relation in the form

$$
\left\{\begin{array}{l}
u_{0}(\Im, \Re, \xi, t)=u(\Im, \Re, \xi, 0), \\
u_{1}(\Im, \Re, \xi, t)=\lambda I_{t}^{\vartheta}\left[-P_{0}-q_{0}-\delta_{0}+\rho_{0}\left(\frac{\partial^{2} u}{\partial \Im^{2}}+\frac{\partial^{2} u}{\partial \Re^{2}}+\frac{\partial^{2} u}{\partial \xi^{2}}\right)+g_{1}\right], \\
u_{k}(\Im, \Re, \xi, t)=\lambda I_{t}^{\vartheta}\left[-P_{k-1}-q_{k-1}-\delta_{k-1}+\rho_{0}\left(\frac{\partial^{2} u_{k-1}}{\partial \Im^{2}}+\frac{\partial^{2} u_{k-1}}{\partial \Re^{2}}+\frac{\partial^{2} u_{k-1}}{\partial \xi^{2}}\right)+g_{1}\right] .
\end{array}\right.
$$

Similarly, we also obtain the recursive scheme

$$
\begin{aligned}
& \left\{\begin{array}{l}
v_{0}(\Im, \Re, \xi, t)=v(\Im, \Re, \xi, 0), \\
u_{1}(\Im, \Re, \xi, t)=\lambda I_{t}^{\vartheta}\left[-P_{0}^{*}-q_{0}^{*}-\delta_{0}^{*}+\rho_{0}\left(\frac{\partial^{2} v}{\partial \Im^{2}}+\frac{\partial^{2} v}{\partial \Re^{2}}+\frac{\partial^{2} v}{\partial \xi^{2}}\right)+g_{2}\right], \\
v_{k}(\Im, \Re, \xi, t)=\lambda I_{t}^{\vartheta}\left[-P_{k-1}^{*}-q_{k-1}^{*}-\delta_{k-1}^{*}+\rho_{0}\left(\frac{\partial^{2} v_{k-1}}{\partial \Im^{2}}+\frac{\partial^{2} v_{k-1}}{\partial \Re^{2}}+\frac{\partial^{2} v_{k-1}}{\partial \xi^{2}}\right)+g_{2}\right],
\end{array}\right. \\
& \left\{\begin{array}{l}
\psi_{0}(\mathfrak{I}, \Re, \xi, t)=\psi(\Im, \Re, \xi, 0), \\
\psi_{1}(\mathfrak{I}, \Re, \xi, t)=\lambda I_{t}^{\vartheta}\left[-P_{0}^{\bullet}-q_{0}^{\bullet}-\delta_{0}^{\bullet}+\rho_{0}\left(\frac{\partial^{2} \psi}{\partial \Im^{2}}+\frac{\partial^{2} \psi}{\partial \Re^{2}}+\frac{\partial^{2} \psi}{\partial \xi^{2}}\right)+g_{3}\right], \\
\psi_{k}(\Im, \Re, \xi, t)=\lambda I_{t}^{\vartheta}\left[-P_{k-1}^{\bullet}-q_{k-1}^{\bullet}-\delta_{k-1}^{\bullet}+\rho_{0}\left(\frac{\partial^{2} \psi_{k-1}}{\partial \Im^{2}}+\frac{\partial^{2} \psi_{k-1}}{\partial \Re^{2}}+\frac{\partial^{2} \psi_{k-1}}{\partial \xi^{2}}\right)+g_{3}\right] .
\end{array}\right.
\end{aligned}
$$

Thus we obtain the NAAM terms of equation (78) in the form

$$
\begin{aligned}
& u_{0}(\Im, \Re, \xi, t)=-0.5 \Im+\Re+\xi \\
& u_{1}(\Im, \Re, \xi, t)=-(2.25 \Im) \frac{t^{\vartheta}}{\Gamma(\vartheta+1)}, \\
& u_{2}(\Im, \Re, \xi, t)=(-2.25 \Im+4.5 \Re+4.5 \xi) \frac{t^{2 \vartheta}}{\Gamma(2 \vartheta+1)}
\end{aligned}
$$


Similarly, we also obtain the NAAM terms of equation (79) in the form

$$
\begin{aligned}
& v_{0}(\Im, \Re, \xi, t)=\Im-0.5 \Re+\xi \\
& v_{1}(\Im, \Re, \xi, t)=-(2.25 \Re) \frac{t^{\vartheta}}{\Gamma(\vartheta+1)}, \\
& v_{2}(\Im, \Re, \xi, t)=(4.5 \Im-2.25 \Re+4.5 \xi) \frac{t^{2 \vartheta}}{\Gamma(2 \vartheta+1)} .
\end{aligned}
$$

$\vdots$

We also obtain the NAAM terms of equation (80) in the form

$$
\begin{aligned}
& \psi_{0}(\Im, \Re, \xi, t)=\Im+\Re-0.5 \xi \\
& \psi_{1}(\Im, \Re, \xi, t)=-(2.25 \xi) \frac{t^{\vartheta}}{\Gamma(\vartheta+1)}, \\
& \psi_{2}(\Im, \Re, \xi, t)=(4.5 \Im+4.5 \Re-2.25 \xi) \frac{t^{2 \vartheta}}{\Gamma(2 \vartheta+1)}
\end{aligned}
$$

Thus the NAAM solution becomes

$$
\left\{\begin{array}{l}
u(\Im, \Re, \xi, t)=u_{0}(\Im, \Re, \xi, t)+u_{1}(\Im, \Re, \xi, t)+u_{2}(\Im, \Re, \xi, t)+\cdots \\
v(\Im, \Re, \xi, t)=v_{0}(\Im, \Re, \xi, t)+v_{1}(\Im, \Re, \xi, t)+v_{2}(\Im, \Re, \xi, t)+\cdots \\
\psi(\Im, \Re, \xi, t)=\psi_{0}(\Im, \Re, \xi, t)+\psi_{1}(\Im, \Re, \xi, t)+\psi_{2}(\Im, \Re, \xi, t)+\cdots
\end{array}\right.
$$

By putting the NAAM components we get

$$
\left\{\begin{aligned}
u(\Im, \Re, \xi, t)= & -0.5 \Im+\Re+\xi \pm(2.25 \Im) \frac{t^{\vartheta}}{\Gamma(\vartheta+1)} \\
& +(-2.25 \Im+4.5 \Re+4.5 \xi) \frac{t^{2 \vartheta}}{\Gamma(2 \vartheta+1)}+\cdots \\
v(\Im, \Re, \xi, t)= & \Im-0.5 \Re+\xi \pm(2.25 \Re) \frac{t^{\vartheta}}{\Gamma(\vartheta+1)} \\
& +(4.5 \Im-2.25 \Re+4.5 \xi) \frac{t^{2 \vartheta}}{\Gamma(2 \vartheta+1)}+\cdots \\
\psi(\Im, \Re, \xi, t)= & \Im+\Re-0.5 \xi \pm(2.25 \xi) \frac{t^{\vartheta}}{\Gamma(\vartheta+1)} \\
& +(4.5 \Im+4.5 \Re-2.25 \xi) \frac{t^{2 \vartheta}}{\Gamma(2 \vartheta+1)}+\cdots
\end{aligned}\right.
$$

For the particular $\vartheta=1$, the solution converges to the exact form solution

$$
\left\{\begin{array}{l}
u(\Im, \Re, \xi, t)=\frac{-0.5 \Im+\Re++\xi \pm(2.25 \Im) t}{1-2.25 t^{2}} \\
v(\Im, \Re, \xi, t)=\frac{\Im \pm 0.5 \Re+\xi \pm(2.25 \Re) t}{1-2.25 t^{2}} \\
\psi(\Im, \Re, \xi, t)=\frac{\Im+\Re \pm 0.5 \xi \pm(2.25 \xi) t}{1-2.25 t^{2}}
\end{array}\right.
$$

\section{Conclusion}

In this paper, we applied a newly developed approximate analytical method based on the iterative procedure and the properties of Caputo and Riemann-Liouville integral operators. 
First, we proceeded to the iterative approximation and then used the Liouville integral operator of fractional order with Caputo derivative. The Liouville-Caputo operator provides the approximated term of fractional order. We have solved some linear and nonlinear wellknown fluid flow models known as Naiver-Stocks equations. The approximation through the proposed method provides the closed-form solutions of the suggested models. The nonlinearity in each problem is evaluated effortlessly and effectively. The technique is simple to assess both linear and nonlinear terms of the testing problems. The NAA method has a less and more powerful computational ability to evaluate the closed-form analytical solution. Overall, the method has less computational procedures and simple to apply for both linear and nonlinear physical models. This method is also applicable to other physical phenomena of fractional order.

Acknowledgements

Researchers Supporting Project number (RSP-2020/210), King Saud University, Riyadh, Saudi Arabia

Availability of data and materials

No data were used to support this study.

Competing interests

The authors declare that they have no competing interests.

\section{Authors' contributions}

The main idea of this paper was proposed by UF, HK, FT, EH, DB and HBJ. KM prepared the manuscript initially and performed all the steps of the proofs in this research. All authors read and approved the final manuscript.

\section{Author details}

${ }^{1}$ Department of Mathematics, Abdul Wali khan University Mardan, Mardan, Pakistan. ${ }^{2}$ Department of Mathematics, Near East University TRNC, Mersin 10, Turkey. ${ }^{3}$ Mathematics Department, King Saudi University, Riyadh, Saudi Arabia. ${ }^{4}$ Department of Mathematics, Faculty of Arts and Sciences, Cankaya University, 06530 Ankara, Turkey. ${ }^{5}$ Institute of Space Sciences, Magurele-Bucharest, Romania.

\section{Publisher's Note}

Springer Nature remains neutral with regard to jurisdictional claims in published maps and institutional affiliations.

Received: 29 October 2020 Accepted: 14 January 2021 Published online: 28 January 2021

\section{References}

1. He, J.H.: Nonlinear oscillation with fractional derivative and its applications. In: Int Conf Vibrating Engg 98, Dalian pp. 288-291

2. He, J.H.: Homotopy perturbation technique. Comput. Methods Appl. Mech. Eng. 178, 257-262 (1999)

3. $W u, X ., L a i, D ., L u, H .:$ Generalized synchronization of the fractional-order chaos in weighted complex dynamical networks with nonidentical nodes. Nonlinear Dyn. 69(1-2), 667-683 (2012)

4. Sheng, H., Chen, Y., Qiu, T.: Fractional Processes and Fractional-Order Signal Processing: Techniques and Applications. Springer, Berlin (2011)

5. Nasrolahpour, H.: A note on fractional electrodynamics. Commun. Nonlinear Sci. Numer. Simul. 18(9), 2589-2593 (2013)

6. Veeresha, P., Prakasha, D.G., Baskonus, H.M.: New numerical surfaces to the mathematical model of cancer chemotherapy effect in Caputo fractional derivatives. Chaos, Interdiscip. J. Nonlinear Sci. 29(1), 013119 (2019)

7. Longhi, S.: Fractional Schrödinger equation in optics. Opt. Lett. 40(6), 1117-1120 (2015)

8. Singh, J., Kumar, D., Baleanu, D.: On the analysis of fractional diabetes model with exponential law. Adv. Differ. Equ. 2018(1), 231 (2018)

9. Khan, H., Shah, R., Kumam, P., Baleanu, D., Arif, M.: Laplace decomposition for solving nonlinear system of fractional order partial differential equations. Adv. Differ. Equ. 2020(1), 375 (2020)

10. Shah, R., Khan, H., Baleanu, D., Kumam, P., Arif, M.: A novel method for the analytical solution of fractional Zakharov-Kuznetsov equations. Adv. Differ. Equ. 2019(1), 517 (2019)

11. Ali, I., Khan, H., Shah, R., Baleanu, D., Kumam, P., Arif, M.: Fractional view analysis of acoustic wave equations, using fractional-order differential equations. Appl. Sci. 10(2), 610 (2020)

12. Hilfer, R.: Applications of Fractional Calculus in Physics. Orlando (1999)

13. Kilbas, A.A., Srivastava, H.M., Trujillo, J.J.: Theory and Applications of Fractional Differential Equations (Vol. 204). Elsevier, Amsterdam (2006)

14. Das, S.: A note on fractional diffusion equations. Chaos Solitons Fractals 42(4), 2074-2079 (2009)

15. Navier, C.L.H.: Mémoire sur les lois du mouvement des fluides. Mém. Acad. Sci. Inst. Fr. 6, 389-440 (1822)

16. El-Shahed, M., Salem, A.: On the generalized Navier-Stokes equations. Appl. Math. Comput. 156(1), $287-293$ (2005) 
17. Kumar, D., Singh, J., Kumar, S.: A fractional model of Navier-Stokes equation arising in unsteady flow of a viscous fluid. J. Assoc. Arab Univ. Basic Appl. Sci. 17, 14-19 (2015)

18. Kumar, S., Kumar, D., Abbasbandy, S., Rashidi, M.M.: Analytical solution of fractional Navier-Stokes equation by using modified Laplace decomposition method. Ain Shams Eng. J. 5(2), 569-574 (2014)

19. Ganji, Z.Z., Ganji, D.D., Ganji, A.D., Rostamian, M.: Analytical solution of time-fractional Navier-Stokes equation in polar coordinate by homotopy perturbation method. Numer. Methods Partial Differ. Equ. 26(1), 117-124 (2010)

20. Ragab, A.A., Hemida, K.M., Mohamed, M.S., Abd El Salam, M.A.: Solution of time-fractional Navier-Stokes equation by using homotopy analysis method. Gen. Math. Notes 13(2), 13-21 (2012)

21. Mahmood, S., Shah, R., Arif, M.: Laplace Adomian decomposition method for multi dimensional time fractional model of Navier-Stokes equation. Symmetry 11(2), 149 (2019)

22. Momani, S., Odibat, Z: Analytical solution of a time-fractional Navier-Stokes equation by Adomian decomposition method. Appl. Math. Comput. 177, 488-494 (2006)

23. Birajdar, G.A.: Numerical solution of time fractional Navier-Stokes equation by discrete Adomian decomposition method. Nonlinear Eng. 3(1), 21-26 (2014)

24. Chaurasia, V.B.L., Kumar, D.: Solution of the time-fractional Navier-Stokes equation. Gen. Math. Notes 4(2), 49-59 (2011)

25. Prakash, A., Veeresha, P., Prakasha, D.G., Goyal, M.: A new efficient technique for solving fractional coupled Navier-Stokes equations using q-homotopy analysis transform method. Pramana 93(1), 6 (2019)

26. Singh, B.K., Kumar, P.: FRDTM for numerical simulation of multi-dimensional, time-fractional model of Navier-Stokes equation. Ain Shams Eng. J. 9(4), 827-834 (2018)

27. Thabet, H., Kendre, S., Peters, J.: Travelling wave solutions for fractional Korteweg-de Vries equations via an approximate-analytical method. AlMS Math. 4(4), 1203 (2019)

28. Prakash, A., Kumar, M., Baleanu, D.: A new iterative technique for a fractional model of nonlinear Zakharov-Kuznetsov equations via Sumudu transform. Appl. Math. Comput. 334, 30-40 (2018)

29. Prakash, A., Veeresha, P., Prakasha, D.G., Goyal, M.: A homotopy technique for a fractional order multi-dimensional telegraph equation via the Laplace transform. Eur. Phys. J. Plus 134(1), 1-18 (2019)

30. Kumar, D., Singh, J., Prakash, A., Swroop, R.: Numerical simulation for system of time-fractional linear and nonlinear differential equations. Prog. Fract. Differ. Appl. 5(1), 65-77 (2019)

31. Goyal, M., Baskonus, H.M., Prakash, A.: An efficient technique for a time fractional model of lassa hemorrhagic fever spreading in pregnant women. Eur. Phys. J. Plus 134(10), 482 (2019)

32. Goyal, M., Baskonus, H.M., Prakash, A.: Regarding new positive, bounded and convergent numerical solution of nonlinear time fractional HIV/AIDS transmission model. Chaos Solitons Fractals 139, 110096 (2020)

33. Prakash, A., Prakasha, D.G., Veeresha, P.: A reliable algorithm for time-fractional Navier-Stokes equations via Laplace transform. Nonlinear Eng. 8(1), 695-701 (2019)

34. Prakash, A., Goyal, M., Gupta, S.: A reliable algorithm for fractional Bloch model arising in magnetic resonance imaging. Pramana 92(2), 18 (2019)

35. Prakash, A., Kaur, H.: Analysis and numerical simulation of fractional Biswas-Milovic model. Math. Comput. Simul. 181, 298-315 (2021)

36. Shah, R., Farooq, U., Khan, H., Baleanu, D., Kumam, P., Arif, M.: Fractional view analysis of third order Kortewege-de Vries equations, using a new analytical technique. Front. Phys. 7, 244 (2020). https://doi.org/10.3389/fphy

37. Sontakke, B.R., Shaikh, A.S.: Properties of Caputo operator and its applications to linear fractional differential equations. Int. J. Eng. Res. Appl. 5(5), 22-29 (2015)

\section{Submit your manuscript to a SpringerOpen ${ }^{\circ}$ journal and benefit from:}

- Convenient online submission

- Rigorous peer review

- Open access: articles freely available online

- High visibility within the field

- Retaining the copyright to your article

Submit your next manuscript at $\gg$ springeropen.com 